\title{
Prioritizing surgery during the COVID-19 pandemic: the Quebec guidelines
}

\author{
Marie-Eve Bouthillier, PhD \\ Michel Lorange, PhD \\ Serge Legault, MD \\ Lucie Wade, MSc \\ Joseph Dahine, MD \\ Jean Latreille, MD \\ Isabelle Germain, MD \\ Roger Grégoire, MD \\ Patrick Montpetit, MD \\ Catherine Prady, MD \\ Elise Thibault, MD \\ Vincent Dumez, MSc \\ Lucie Opatrny, MD
}

Accepted January 12, 2021

\section{Correspondence to:}

M.E. Bouthillier

Faculty of Medicine

Université de Montréal

C. P. 6128 , succursale Centre-ville

Montréal, QC H2R 2G7

bouthillier@me.com

DOI: 10.1503/cjs.022220

\section{SUMmARY}

In many countries, health care institutions have ramped down nonemergent activities in order to free up hospital and critical care beds in anticipation of a wave of patients with coronavirus disease 2019 (COVID-19). Medical activities were reduced to a minimum, leaving operating rooms to run semiurgent and urgent surgeries only. The status quo of systematically prioritizing resources away from surgical care to patients with COVID-19 may lead to unintended long-term outcomes. We propose a 4-step prioritization system based on resource availability and clinical criteria, as well as supplemental triage criteria for instances where multiple patients have equal claims to priority. The algorithm aims to guide clinicians and decision-makers toward allocating resources to surgical patients while still optimizing pandemicspecific benefits to the population.

$\mathbf{T}$ he coronavirus disease 2019 (COVID-19) pandemic has had a major effect on the ability of health care institutions to continue to provide comprehensive access to surgery. In March 2020, the ministry of health in Quebec, Canada (Ministère de la Santé et des Services Sociaux), created a COVID-19 clinical steering committee to imagine and implement the necessary reorganization of the health care system. The ministry asked institutions to ramp down nonemergent activities to free up a large number of hospital and critical care beds in anticipation of a wave of patients with COVID-19. Surgical activities were reduced to $60 \%-70 \%$ of normal capacity. All elective surgeries were cancelled, leaving operating rooms (ORs) to run only semiurgent and urgent surgeries. A working group, which the authors represent, was implemented to consider the ethical issues around access to surgery and provide national guidelines for prioritizing surgeries during this exceptional context. Our group aimed to answer 2 questions. First, what patients should have access to surgery when resources are limited? Second, how should we ensure an equitable distribution of resources to maximize the benefits to the entire population?

\section{Process}

A literature review served as the basis for discussion. The criteria of the prioritization system were identified by consensus. Those involved in the process were surgeons $(n=5)$, radiation oncologists $(n=1)$, oncologists $(n=1)$, the national director of a cancer program, patients $(n=6)$, intensivists $(n=1)$, ethicists $(n=3)$, and research professionals $(n=2)$. These 20 people were divided into 3 groups, including a working group that developed a first draft of the system $(n=9)$, a review group that revised and enhanced the system to a final version $(n=5)$ and a patient group $(n=6)$ that also reviewed and commented on the draft. Several iterations were necessary before achieving consensus among all groups. 


\section{THE PRIORITIZATION SYSTEM}

The system aims to support the orientation of each patient. Its ethical principles are benefit maximization, proportionality, nonmaleficence, equity, reciprocity, agility and protection of stakeholders and the public. Continuous communication between the surgeon and the patient transcends this system. Continuous follow-up is required and provides an opportunity to reassure and respond to the questions and concerns of patients and family members. ${ }^{1}$ The system contains 4 sequential steps based on resource availability, clinical criteria and supplemental triage criteria when needed as tie-breakers.

\section{Step 1: maximize the daily capacity of the OR}

Institutions determine their daily OR capacity. They negotiate an equitable distribution of resources, aiming to maximize benefits, proportional to current demand, across patient populations. Once agreement is reached on the distribution of resources, the OR capacity is finalized (Figure 1).

\section{Step 2: pretriage by specialty}

Individual surgeons within each specialty pretriage all their patients. The goal of pretriage is to identify patients for whom the surgery is essential and for whom alternative care is not an acceptable equivalent (Figure 2).

\section{Step 3: overall prioritization}

Final prioritization of surgical cases across specialties is a dynamic and continuous process that adapts to various changes in OR capacity and patient condition. First, a list is produced by each surgeon within each specialty. Second, a single list combines all the patients (regardless of surgeon) within each specialty.

Decisions are then made by a local committee that applies the proposed prioritization criteria across specialties (Figure 3 ). This algorithm proposes more stringent clinical criteria as capacity decreases, but demands frequent reassessment as resources become available again, until the backlog is empty. If 2 or more patients have equal claims to priority, 2 supplemental criteria are proposed to choose fairly. The "fair innings" criterion favours intergenerational equity and therefore gives priority to the person who is at an earlier stage of life. ${ }^{2}$ If this criterion is not applicable, randomization will be carried out to best respect equality of opportunity between patients.,

\section{Step 1: determine the capacity of the operating room}



Fig. 1. Step 1: determine the capacity of the operating room. 


\section{Step 2: pretriage of patients}

Does the level of care

and the patient's

preferences necessitate

a surgery?

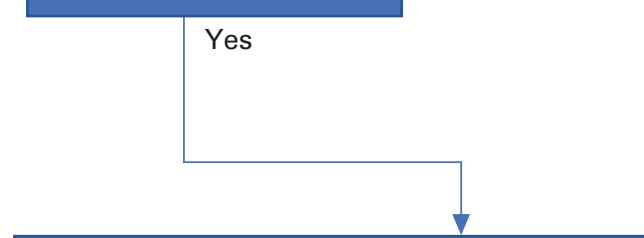

Can the patient obtain an alternative treatment? Evaluate whether the patient should remain on the waiting list.

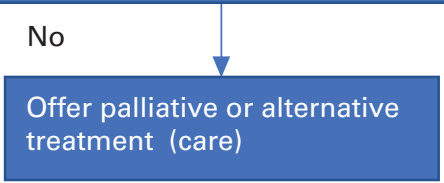

Is surgery essential in the short term?

a. Threat to patient's immediate survival if surgery is not performed

b. Threat of permanent limb or organ dysfunction

c. Rapidly evolving neoplasm

d. Risk of progression of the disease (reduction of possibility of recovery or more important intervention later, more morbid treatment, risk of inoperability)

e. Risk of worsening toward more severe symptoms, consuming more pain medication or developing chronic pain because of delay in treatment

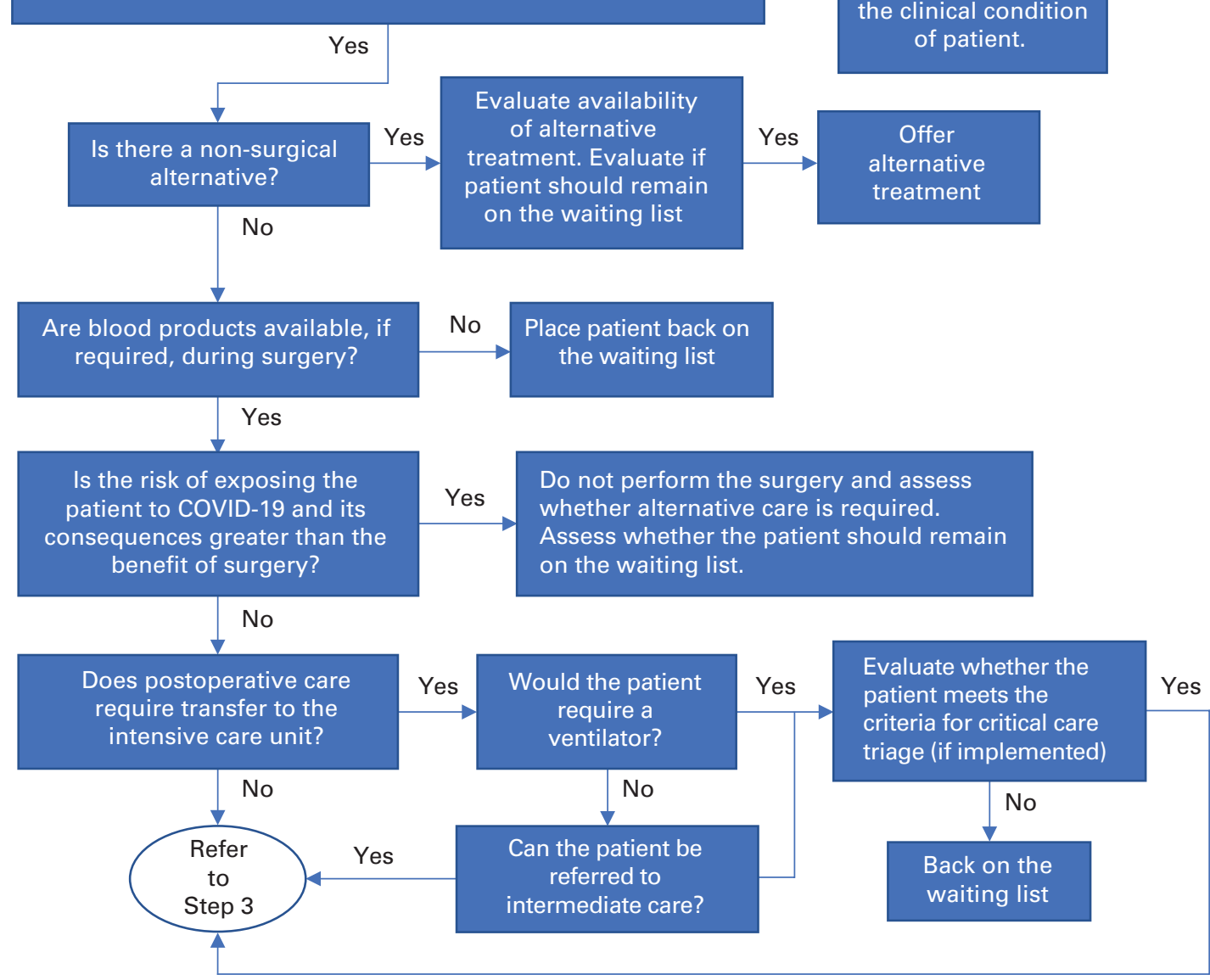

Fig. 2. Step 2: pretriage of patients, by specialty. Alternative treatment refers to a treatment that may not be the best curative option, but an alternative to the standard of care. Intermediate care refers to care units for patients who are more stable than those in intensive care, but who require specialized care that is not available in acute care medical and surgical units. Palliative care refers to care focused on the patient's quality of life, without the objective of curing the patient. COVID-19= coronavirus disease 19 . 
Step 3: overall prioritization of patients awaiting surgery

The prioritization is done in two stages using the same decision-making process:

- By specialty

- All specialties combined

Ensure that the patient

list considers the capacity

of other clinical services

e.g., radiotherapy,

rehabilitation

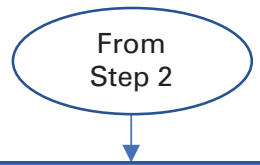

$30 \%$ to $50 \%$ of the operating room capacity

Prioritize patient according to:

- Those whose survival is most at risk

- Those nearest to or beyond the maximum surgery date

- Those requiring the least amount of operating

time and resources possible (considering the cases most at risk and requiring more operating time.)

- Those most likely to recover quickly (low ASA classification)

$50 \%$ to $70 \%$ of the operating room capacity

Prioritize patients according to:

- Those whose survival is most at risk

- Those nearest or closest to the maximum surgery date

- Those requiring the least amount of operating time and resources possible

- Those with good chance to recover quickly (low to medium ASA classification)

$70 \%$ to $100 \%$ of the operating room capacity

When operating room resources increase, add $10 \%$

of less urgent patients per week based on their wait

time for surgery.

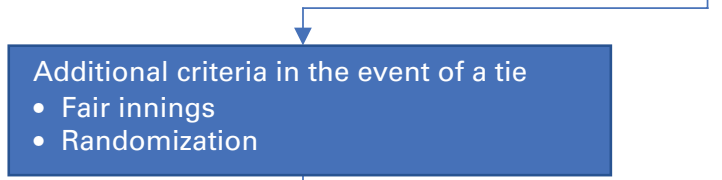

Priority list of patients

for all specialties (with

resources required for

each patient)

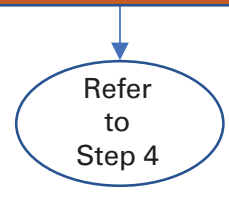

Fig. 3. Step 3: overall prioritization of patients awaiting surgery. ASA = American Society of Anesthesiologists.

\section{Step 4: determining the OR schedule}

The operating schedule is constructed by combining the daily OR capacity (step 1) and the prioritized lists of patients (result of step 2 and 3) (Figure 4).

\section{ReCommendations}

This prioritization system can only be justified on the basis of equity if it is applied in the larger context of prioritizing the care needs of the entire population. To that 


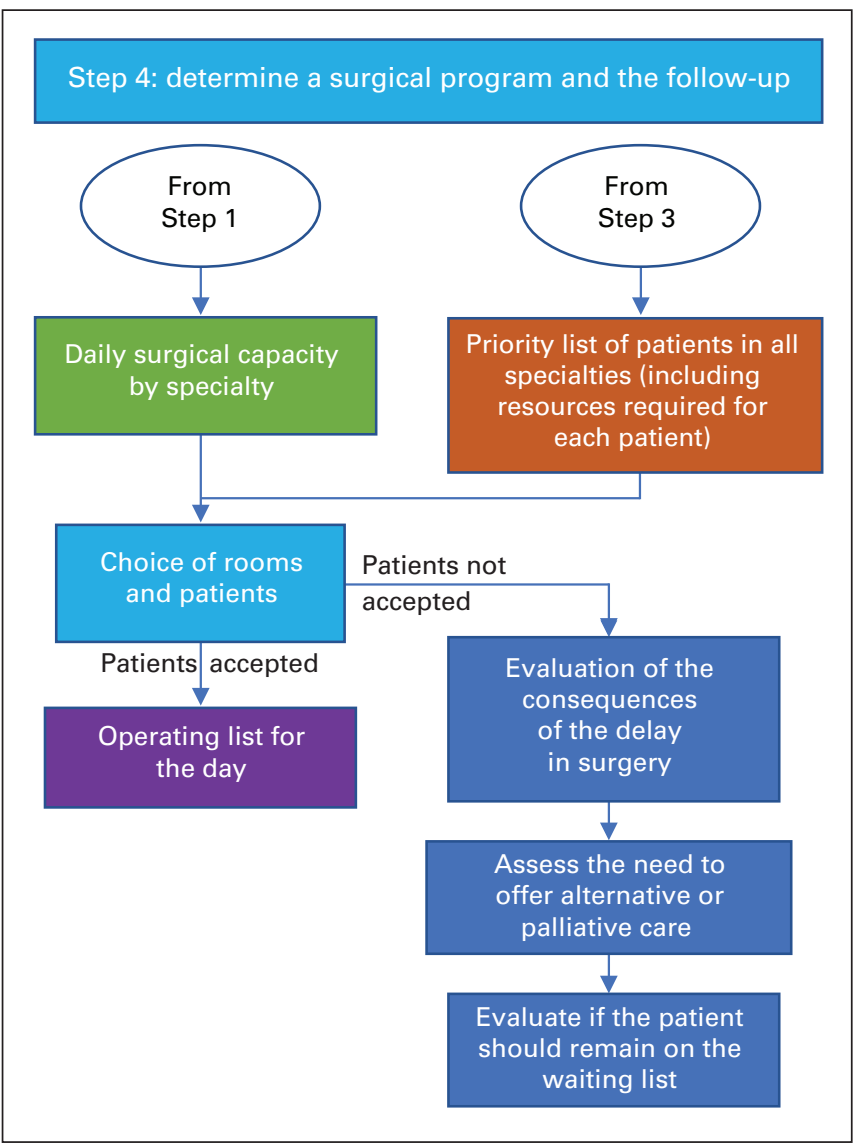

Fig. 4. Step 4: determining the operating room schedule.

end, 3 recommendations emerge for maximizing population benefit during the COVID-19 pandemic. First, a system for prioritizing access to surgery during a pandemic should be applied immediately to identify patients across specialties who should have access to surgery, even when resources are limited. Otherwise, people suffering from life-threatening clinical conditions other than COVID-19 may be disproportionately disadvantaged, resulting in indirect morbidity and deaths from the pandemic beyond those caused by COVID-19 directly. Second, implementation of this protocol should be uniform across jurisdictions so that the entire population can have equitable access to scarce resources. Finally, many patients will not be selected and will continue to wait for their surgery, most likely with increasing anxiety and stress over time. Maintenance of the therapeutic relationship between patients and surgeons is important to assess the clinical and emotional consequences of delays.

\section{Conclusion}

Our group advocates for the regionalization of resource allocation. This can be achieved with standardized surgical triage criteria based on clinical relevance. We submit that such a system will minimize the harm of the COVID-19 pandemic to the countless patients on surgical waiting lists.

Affiliations: From the Faculty of Medicine, Université de Montréal, Montréal, Que. (Bouthillier, Dumez); the Centre intégré de santé et de services sociaux de Laval, Laval, Que. (Lorange, Dahine, Montpetit); the Ministère de la Santé et des Services Sociaux, Que. (Legault, Latreille, Opatrny); McGill University Health Centre, Montréal, Que. (Wade); the National Radiation Oncology Committee, Que. (Germain); the Centre Hospitalier Universitaire de Québec, Québec, Que. (Grégoire); the National Breast Cancer Committee (Prady); the Quebec Association of Vascular and Endovascular Surgery (Thibault); the Centre Hospitalier Universitaire de Montréal, Montréal, Que. (Dumez).

Competing interests: None declared.

Contributors: All authors contributed substantially to the conception, writing and revision of this article and approved the final version for publication.

Content licence: This is an Open Access article distributed in accordance with the terms of the Creative Commons Attribution (CC BY-NC-ND 4.0) licence, which permits use, distribution and reproduction in any medium, provided that the original publication is properly cited, the use is noncommercial (i.e., research or educational use), and no modifications or adaptations are made. See: https://creativecommons.org/licenses/by-nc-nd/4.0/

\section{References}

1. Système de priorisation pour l'accès à une chirurgie en situation de pandémie. [En ligne]. Ministère de la Santé et des Services Sociaux: Québec; April 2020. Available: https://www.msss. gouv.qc.ca/professionnels/documents/coronavirus-2019-ncov/ PJ1_Systeme_priorisation_acces_chirurgie-pandemie_v4-4.pdf (accessed 2020 Oct. 24).

2. Biddison LD, Gwon HS, Schoch-Spana M, et al. Scarce resource allocation during disasters. a mixed-method community engagement study. Chest 2018;153:187-95.

3. Winsor S, Bensimon CM, Sibbald R, et al. Identifying prioritization criteria to supplement critical care triage protocols for the allocation of ventilators during a pandemic influenza. Healthc $Q$ 2014;17:44-51.

4. Farmer Y. La distribution aléatoire des ressources en santé: pour un modèle hybride équilibrant les principes de justice et de maximization. Can f Public Health 2012;103:119-21. 